\title{
EDITORIAL
}

\section{Wer hat Angst vor Jean-Paul Sartre?}

In einem Gespräch mit Paul Ricœur im Jahr 2003 kamen wir auf Sartre zu sprechen. Da sagte er auf einmal aufgeregt: "Wussten Sie, dass Sartre nach seinem Tod bei uns in Ungnade gefallen ist? Er wurde verboten, seine Philosophie kommt deshalb im akademischen Diskurs kaum mehr vor. Er wird an den Universitäten nicht mehr unterrichtet und man kann über ihn auch kaum mehr dissertieren". Die Frage "Wussten Sie...?", deutete darauf hin, dass diese Tatsache nicht unbedingt bekannt war. Einer der wenigen, der darauf aufmerksam machte, war Bernard-Henry Lévy:

Meine Lehrmeister (...) waren mittlerweile selbst verstorben. Aber das Verbotene [sic!] hielt sich hartnäckig. Im literarischen Fundus des Zeitgeistes galt die Sartresche Maske zweifellos als der Ladenhüter, dasjenige, wonach am wenigsten gefragt wurde (Lévy 2002, 14).

Wenn heute Sartres Werk von seinen Gegnern als uninteressant und vergessen dargestellt wird, mit dem Hinweis, man lese seine Werke in Frankreich kaum mehr, so wird dieses Verbot natürlich verschwiegen, ohne darauf einzugehen, warum er in anderen Länder doch noch gelesen und über ihn geforscht wird. Als ich im Jahr 2005 eine internationale Konferenz zum 100. Todestag von Sartre an der Universität Wien organisierte, lehnte das Französische Kulturinstitut, im Gegensatz zur Kanadischen Botschaft, energisch ab die Konferenz zu unterstützen. Die Frage, die sich deshalb immer wieder aufdrängt lautet: Wer hat Angst vor Jean-Paul Sartre? Was hat ihn so unbequem gemacht, dass man nach seinem Tod jede Erinnerung an ihn "auslöschen" wollte (siehe Enthoven 2005)? Warum sind die Meinungen auch heute noch so gespalten, sobald sein Name fällt?

Um diese Frage zu klären bräuchte man lediglich die Vorwürfe in Betracht zu ziehen, die in den letzten 35 Jahren immer aufs Neue formuliert wurden. Sartre, so sagt man, sei vielleicht ein guter Schriftsteller und ein großer Philosoph gewesen, aber er habe sich in allen politischen Fragen geirrt, er sei auf der falschen Seite gestanden, derjenigen der Kommunisten, im Gegensatz zu Raymond Aron, der zwar nie zum "Genie" erhoben wurde, aber dafür politisch "die richtige Position", die des Westens, vertreten habe. "Es wäre besser und im Interesse von Sartre, der ein wichtiger Philosoph und Schriftsteller ist, den politisierenden Sartre zu vergessen, der sich lächerlich macht, in dem er Stalin, Mao und Castro bewundert" - teilte zum 100. Sartre-Jubiläum der Aron-Schüler Jean-Claude Casanova der 
Zeitschrift Express mit (Casanova 2005). Und so muss jedes Mal von Neuem erklärt werden, dass Sartres Verteidigung der Kommunisten in den 50er Jahren durch den kalten Krieg bedingt war, in dem die USA die Übermacht besaßen. Sartre deklarierte in "Les communistes et la paix", dass sein Einverständnis mit den Kommunisten begrenzt sei und dass er keineswegs alle ihre Prinzipien teile. ${ }^{1}$ Nach der russischen Invasion in Ungarn 1957 brach er überhaupt die Kontakte mit der UDSSR ab, verurteilte die sowjetischen Lager (Sartre 1956), setzte sich für die russischen Dissidenten ein und wurde von Chruschtschow beschuldigt, Antikommunist und Gehilfe des Kapitalismus zu sein. Sartre war auch nicht blind gegenüber der Politik Mao Tse Tungs: er verurteilte die chinesischen Lager und hob die Bedeutung des Berichts Prisonnier de Mao von Jean Pasqualini hervor, der seine siebenjährige Gefangenschaft in einem chinesischen Arbeitslager ausführlich beschrieb (Sartre 1976, 221).

Andere, wie zum Beispiel Peter Sloterdijk, sehen in Sartre nur einen "großen Verneiner" (Sloterdijk 2005). Doch Sartres Kritik, die sich gegen alle Formen der Unterdrückung richtet, hat ein positives Ziel, nämlich den "integralen Humanismus". Sartres synthetische Anthropologie und seine Konversionsauffassung bauen auf dem positiven Ansatz einer Erlösung "ohne Gott" auf. Auch seine Auseinandersetzungen mit dem Politischen und seine Europa-Vision zielen letztendlich auf eine axiologische Begründung ab, die von der Idee der kreativen Erschaffung neuer Gemeinschaften, Lebensformen und Werte geleitet ist.

Sartres Kritik an der Moderne stellt alle universalistischen Systeme, aprioristischen Wertordnungen und abstrakten Menschenrechte in Frage. Im zweiten Band der Kritik der dialektischen Vernunft werden die zwei entgegengesetzten gesellschaftlichen Modelle der Moderne hinterfragt: der Kapitalismus mit seiner bürgerlichen Demokratie als eines nichtzentralistischen Systems und der sowjetische Sozialismus als ein bürokratisches und totalitäres System. Da Sartre überzeugt war, dass beide Modelle in sich Mechanismen bergen, die zur Unterdrückung und Entfremdung des Individuums führen, konnte er sich mit keinem der beiden anfreunden. Schon in den 40er Jahren war er davon überzeugt, dass man in Europa nach neuen Lösungen suchen sollte:

Der historisch Handelnde ist fast immer jemand, der angesichts eines Dilemmas plötzlich eine dritte, bis dahin unsichtbare Möglichkeit auftauchen lässt. Zwischen der

\footnotetext{
${ }^{1}$ Zur Erinnerung: "Il est vrai, le but de cet article est de déclarer mon accord avec les communistes sur des sujets précis et limités, en raisonnant à partir de mes principes et non des leurs. (...) Il est arrivé cent fois, depuis le Congrès de Tours, que des hommes ou des groupes 'de gauche' proclament leur accord de fait avec le P. C. tout en soulignant leurs divergences de principe" (Sartre 1964, 168; Siehe auch Birkhall 2004, 146).
} 
Sowjetunion und dem angelsächsischen Block muß man zwar wählen. Ein sozialistisches Europa dagegen ist nicht 'zu wählen', da es ja nicht existiert: es ist zu schaffen (Sartre 1986, 224).

Vor diese Aufgaben sind wir heute erneut gestellt, in einem Europa, das immer noch nicht zu sich selbst gefunden hat, das mehr denn je fragil, sozial und politisch gespalten, ökonomisch instabil und nach den Angriffen auf Charlie-Hebdo und der Flüchtlingsflut zum Teil auch "ratlos" da steht, kurz - ein Europa, das dringend neu zu denken und insgesamt neu zu schaffen ist, wenn es nicht als solches vor unseren Augen untergehen soll.

$$
* * *
$$

Wir haben die beiden Nummern der Zeitschrift Labyrinth im Jahr 2015 Sartres 110. Geburtstagsjubiläum gewidmet und in zwei große Themenkreise unterteilt: Band 1 umfasst das kritische Denken Sartres und Band 2 ist dem Bezug zwischen geschichtlicher Situiertheit und individueller Wahl gewidmet. Einige der hier veröffentlichten Beiträge wurden an der Tagung, die ich anlässlich seines 100. Geburtstags an der Universität Wien organisiert habe, gehalten. Sie wurden später überarbeitet und erscheinen hier in einer neuen Fassung. Andere sind wiederum speziell für die jetzige Ausgabe von Labyrinth verfasst worden. Eine Ausnahme davon und einen besonderen Stellenwert, zumindest für mich, kommt dem Artikel des verstorbenen Kollegen und Freundes Lars Andrée (1950-2003) zu. Er hat, auf meine Einladung hin, diesen für einer internationalen Tagung im Jahr 1994 gehalten. Der Aufsatz erschien ein wenig später in bulgarischer Übersetzung in Filosofski alternativi (1995/4). Hier wird er im französischen Original veröffentlicht, als einer Art verspätete Hommage an den schwedischen Kollegen, der sich intensiv mit Sartres Philosophie- und Literaturwerk beschäftigte. Herzlichen Dank an Alle, die zu dieser neuen Ausgabe beigetragen haben. 


\section{Literaturangaben}

Birchall, Ian H. Sartre against Stalinism. New York / Oxford: Berghan Books, 2004.

Casanova, Jean-Claude / Contat, Michel, "Deux êtres et le réel", propos recueillis par Thierry Gandillot et Marie Zawisza, L'Express, 28.02.2005 (online - http://www.lexpress.fr/culture/livre/deuxetres-et-le-reel_826622.html)

Enthoven, Raphä̈l. "Il reste à tuer l'image de Sartre". Le Nouvel Observateur, le mardi 21 juin 2005 (online: http://tempsreel.nouvelobs.com/culture/20050621.OBS0909/il-reste-a-tuerl-image-desartre.html)

Lévy, Bernard-Henry. Sartre. Der Philosoph des 20. Jahrhunderts. München/Wien: Hanser Verlag, 2002.

Sartre, Jean-Paul. "Après Budapest, Sartre parle", L'Express, supplément au No. 281, 9 novembre 1956; repris dans Michel Contat / Michel Rybalka (ed.). Les écrits de Jean-Paul Sartre. Paris: Gallimard, 1970, 304-306.

Sartre, Jean-Paul. "Les communistes et la paix", in Situations, VI. Problèmes du marxisme, 1. Gallimard, 1964, 80-384.

Sartre, Jean-Paul. "Autoportrait à soixante-dix ans", in: Situations X, Paris: Gallimard, 1976, 133-226.

Sartre, Jean-Paul. Was ist Literatur? Reinbek bei Hamburg: Rowohlt 1986.

Sloterdijk, Peter. "Le grand négateur", Le Nouvel Observateur, jeudi 3 mars 2005 - n²104 (repris dans Traverses, le mardi 14 avril 2009 - http://www.deligne.eu/traverses/sloterdijk-negateur.html) 